\title{
Phonon-drag thermopower in graphene at low temperatures
}

\author{
Mohd Meenhaz Ansari $^{1 *}$, SSZ Ashraf ${ }^{2}$ and Afzal Ahmad ${ }^{1}$ \\ ${ }^{1}$ Applied Physics Department, Zakir Husain College of Engineering \& Technology \\ Aligarh Muslim University Aligarh-202002, Uttar Pradesh, India \\ ${ }^{2}$ Physics Department, Faculty of Science, \\ Aligarh Muslim University, Aligarh-202002, Uttar Pradesh, India \\ *Email: meenhazphysics@gmail.com
}

We investigate the energy dependent thermopower of hot carriers in graphene through coupling to acoustic phonons interacting via the deformation potential in the Boltzmann transport equation approach. We incorporate the full unscreened numerical computation of the equations in the estimation of the phonon-drag thermopower as the analytical result reported in the literature has been deduced neglecting chirality which has a more functional role in graphene. It is observed that on the incorporation of full unscreened numerical approach, the magnitude as well as the power exponent both is affected with the power exponent changed from $\mathrm{T}^{3}$ to $\mathrm{T}^{3.30}$ in the lower temperature range up to $6 \mathrm{~K}$ and nonlinear dependence in the higher temperature range (Figure 1).

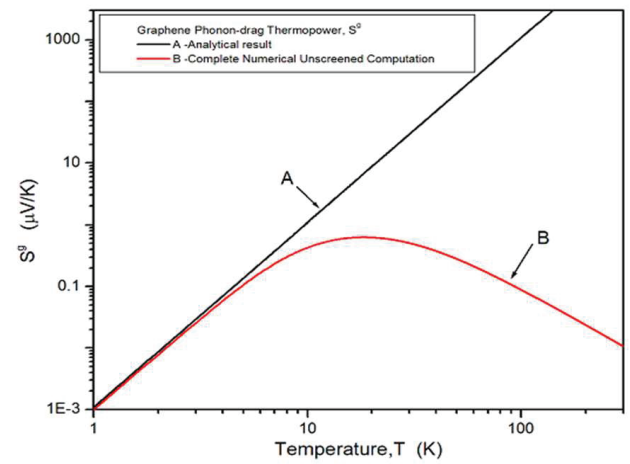

Figure 1: Phonon-drag Thermopower behavior as a function of Temperature. Curve $\mathrm{A}$ is the analytical result and curve $\mathrm{B}$ is the full numerical unscreened computation of the thermopower equation.

We also determine the thermopower behavior as a function of concentration. We find that with increasing concentration, the thermopower reduces at lower temperatures but after a limit, the thermopower is found to increase with concentration (Figure 2). This interestingly result might be the indication of phase transformation in the material.

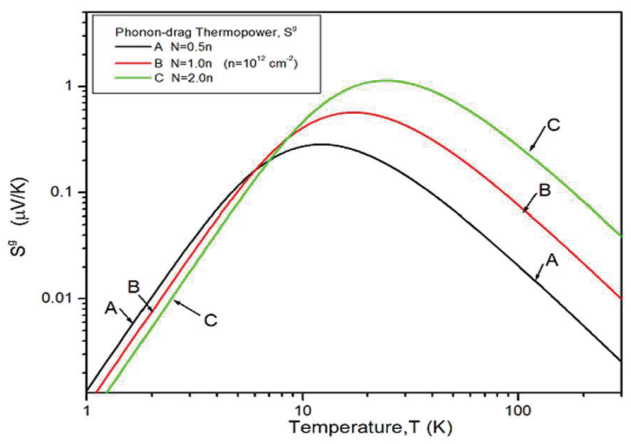

Figure 2: Phonon-drag Thermopower behavior as a function of Concentration. Curve $A$ is for $N=0.5 n$ and curve $\mathrm{B}$ is for $\mathrm{N}=1.0 \mathrm{n}$ and curve $\mathrm{C}$ is for $\mathrm{N}=$ 2.0n where $\mathrm{n}=10^{12} \mathrm{~cm}^{-2}$.

\section{References}

1. A. Castro, K. Novoselov, A.K. Geim etal, Rev. Mod. Phys. 81, (2009), 109.

2. Yuri Zuev, W. Chan and P. Kim, Phys. Rev. Lett. 102, (2009), 096807.

3. S.S. Kubakaddi, Phys. Rev. B, 79, (2009), 075417.

4. S.S. Kubakaddi and K.S. Bhargavi, Phys. Rev. B, 82, (2010), 155410.

5. E.H. Hwang, S. Adam and S. Das Sarma, Phys. Rev. Lett. 98, (2007), 186806.

6. V. W. Scarola and G.D. Mahan, Phys. Rev. B, 66, (2002), 205405.

7. T. Lofwander and M. Fogelstrom, ibid. 76, (2007), 193401. 